\title{
Significant energy relaxation of quantum dot emitted hot electrons
}

\author{
Dongsung T. Park $\odot,{ }^{1}$ Uhjin Kim, ${ }^{2}$ Dongkun Kim, ${ }^{2}$ Hwanchul Jung, ${ }^{3}$ Juho Choi $\odot,{ }^{1}$ Cheolhee Han, ${ }^{1}$ Yunchul Chung $\odot,{ }^{3}$ \\ H.-S. Sim, ${ }^{1}$ V. Umansky, ${ }^{4}$ Hyoungsoon Choi, ${ }^{1,}{ }^{*}$ and Hyung Kook Choi $\odot^{2, \dagger}$ \\ ${ }^{1}$ Department of Physics, KAIST, Daejeon 34141, Republic of Korea \\ ${ }^{2}$ Department of Physics, Research Institute of Physics and Chemistry, Jeonbuk National University, Jeonju 54896, Republic of Korea \\ ${ }^{3}$ Department of Physics, Pusan National University, Busan 46241, Republic of Korea \\ ${ }^{4}$ Department of Condensed Matter Physics, Weizmann Institute of Science, Rehovot 76100, Israel
}

(Received 23 April 2020; revised 23 April 2021; accepted 2 June 2021; published 2 July 2021)

\begin{abstract}
Mesoscopic quantum dots (QDs) are ubiquitous in quantum devices as reliable sources of hot electrons. However, we have observed an unexpectedly significant energy relaxation of QD-emitted hot electrons up to $\approx 55 \%$ of its excitation $\leqslant 1.5 \mathrm{meV}$ from the Fermi level. The energetics of hot electrons were obtained through transverse magnetic focusing over a few microns using both QD and quantum point contact (QPC) emitters. Unlike the QPC counterparts, QD emissions deviated substantially from Fermi gas predictions-the focusing peak appeared at lower magnetic fields, and excessive broadening was observed. The phenomenon was modeled by a capacitive interaction transferring energy from the hot electron to the QD. Model simulations reproduced the key experimental features, implying the presence of a strong yet overlooked relaxation mechanism that is intrinsic to QD emissions. Our observation calls for the prudent use of QDs as single electron sources.
\end{abstract}

DOI: 10.1103/PhysRevResearch.3.033015

\section{INTRODUCTION}

Condensed matter physics provides a powerful framework for understanding the vast array of macroscopic systemsband metals, superconductors, and topological insulators, just to list a few [1-3]. The complexity of describing an almost infinite many-body system is greatly reduced by considering nearly independent quasiparticles, emergent quantum excitations which act as free particles [4]. Naturally, the properties of quasiparticles are a fundamental topic in the study of modern quantum phenomena [5]. In solids, generic fermionic quasiparticles are often alluded to as electrons, and their excitations, also known as hot electrons, serve as an archetype of fermionic excitations.

The quantum coherence of these quasiparticles lends them as a resource in mesoscopic device applications, such as flying qubits and single electron sources [6-8]. The coherence timescale is limited by various relaxation processes, but relaxation phenomena have proven to be a complicated topic with diverse mechanisms and still an active field of research [9-17]. For example, recent studies in quantum Hall systems have shown that hot electrons emitted from quantum dots (QDs) experience drastic energy relaxations within ultrashort length scales $<1 \mu \mathrm{m}$ [17]. Said results are distinguished from those observed in the conventionally studied quantum point contact (QPC) $[18,19]$. Although one may generally expect that QD-

\footnotetext{
*h.choi@kaist.ac.kr

†hkchoi@jbnu.ac.kr

Published by the American Physical Society under the terms of the Creative Commons Attribution 4.0 International license. Further distribution of this work must maintain attribution to the author(s) and the published article's title, journal citation, and DOI.
}

and QPC-emitted hot electrons differ in their relaxations, the reasons as to how much and why the differences emerge have yet to be fully understood. Such characteristics are especially important at low magnetic fields, where a wide range of device applications would realistically operate.

Here, we report the observation of strong energy relaxation in two-dimensional (2D) QD-emitted hot electrons at low magnetic fields. Using the energy scaling properties of transverse magnetic focusing (TMF) [20-23], the energetic mean and standard deviation were obtained for both QD- and QPCemitted hot electrons with initial excitations ranging from 0 to $1.5 \mathrm{meV}$. The QD was carefully tuned to transmit only through a single level in the intermediate-coupling regime; the QPC, in the single-mode bidirectional transport regime for the accuracy and precision required for comparative analysis [24]. While QPC hot electrons showed good agreement with Fermi gas predictions, QD hot electrons exhibited large energy loss and excessive energy broadening which increased with excitations. Surprisingly, the energy relaxation was insensitive to changes in the focusing path lengths in the range $1.5-3 \mu \mathrm{m}$. The length-independent energy relaxation was modeled by a capacitive interaction between the QD and the 2D reservoir, reflecting the electric disturbances created from charge fluctuations during charge transmission. The model simulation presented similar energy statistics and produced a TMF spectrum analogous to experimental measurements, indicating that QD hot electrons can be significantly relaxed immediately after emission by exciting the electrons left in the QD.

\section{DEVICE AND METHODS}

\section{A. Experimental device and measurement setup}

Figure 1(a) represents the nominal device measured in this paper. Electrons from the source reservoirs [Fig. 1(a) red 


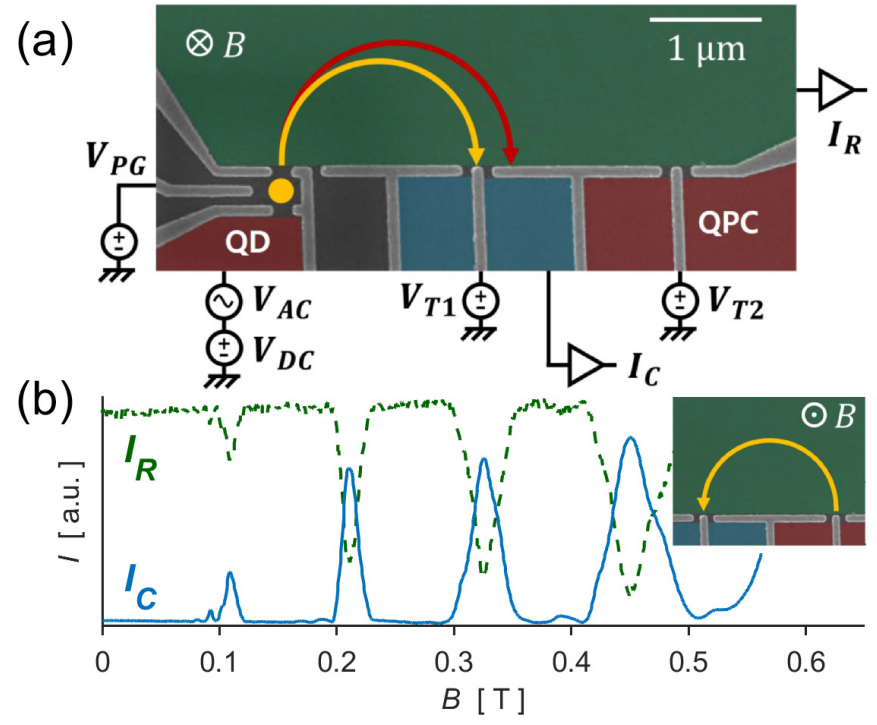

FIG. 1. Quantum dot (QD)-emitter device and typical transverse magnetic focusing (TMF) spectrum. (a) False-colored scanning electron micrograph of the device. A QD and two trench-gated quantum point contacts (QPCs) were defined on a GaAs/AlGaAs heterostructure. The QD or the right QPC was used as the emitter (red reservoirs) and the center QPC as the collector (blue reservoir). The measurement scheme is shown for a QD-emitter experiment. Focused electrons (yellow line) are drained past the collector $\left(I_{C}\right)$, while the rest are drained from the open reservoir $\left(I_{R}\right.$, green area). Electrons with higher energies are focused further away (red line). (b) In a typical QPC TMF spectrum, peaks in $I_{C}$ (solid blue) and dips in $I_{R}$ (dashed green) appear at multiples of $B_{f}$. The right QPC was used for the QPC-emitter experiment (inset).

areas] were transmitted through a QD or QPC emitter. The electron beams traveled through the open reservoir [Fig. 1(a) green area], then were either drained through the collector reservoir [Fig. 1(a) blue area, $I_{C}$ ] or reflected back into the open reservoir $\left(I_{R}\right)$. The Fermi level of source reservoirs was modulated by applying a voltage bias $V_{\mathrm{DC}}$ and understood to be $E_{\mathrm{DC}}=-e V_{\mathrm{DC}}$ above the Fermi energy $E_{F} \approx 7.4 \mathrm{meV}$. The plunger gate voltage $V_{\mathrm{PG}}$ was used to modulate the QD energy levels.

The experimental devices were fabricated on a GaAs/AlGaAs heterojunction containing a 2D electron gas (2DEG) lying $75 \mathrm{~nm}$ under the surface with density $n=2.08 \times 10^{11} \mathrm{~cm}^{-2}$ and mobility $\mu=3.8 \times 10^{6} \mathrm{~cm}^{2} / \mathrm{Vs}$, corresponding with a Fermi energy of $E_{F} \approx 7.4 \mathrm{meV}$ and mean-free path $\approx 29 \mu \mathrm{m}$. Metallic Schottky gates of $75 \mathrm{~nm}$ widths were deposited on the surface using standard electron beam lithography. A QD was formed by depleting the 2DEG underneath four neighboring gates. The QPCs were defined using three gates rather than the traditional two; a trench gate screened the electric field of the split gates and sharpened the confinement potential, thereby widening the subband energy spacing and allowing the QPC to retain its conductance quantization for a wider range of bias voltages [25,26]. An alternating current (AC) excitation of $V_{\mathrm{AC}} \leqslant 10 \mu \mathrm{V}_{\mathrm{rms}}$ at $987.6 \mathrm{~Hz}$ and a direct current (DC) voltage bias $V_{\mathrm{DC}}$ were summed through a bias tee then supplied to the relevant

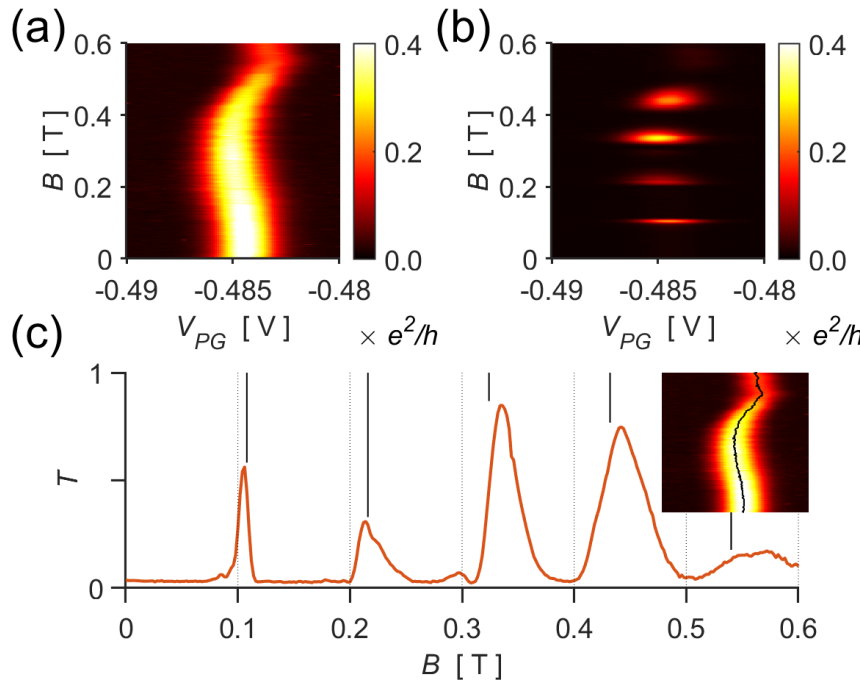

FIG. 2. Quantum dot (QD)-emitter transverse magnetic focusing (TMF) spectrum at equilibrium. (a) QD conductance $\left(I_{C}+I_{R}\right) / V_{A C}$ shows the Coulomb blockade peak shifting erratically with the magnetic field. (b) Conductance through the collector $I_{C} / V_{\mathrm{AC}}$ is the product of the focusing spectrum and QD conductance. (c) $I_{C}$ traced along the peak (inset) gives us the TMF transmission $T$. Finite widths of the QD and QPC emitters result in a slight mismatch between the observed $B_{f}$ (solid black lines) and the expected values (dashed gray lines).

source reservoirs. The currents drained at the collector $\left(I_{C}\right)$ and open reservoir $\left(I_{R}\right)$ were measured simultaneously using the lock-in technique with homemade transimpedance preamplifiers [27]. Two types of devices were fabricated with different distances between the emitters and collector: 1 and $1.5 \mu \mathrm{m}$. Figures 1 and 2 feature a $1.5 \mu \mathrm{m}$ device, tested at an electron temperature of $\approx 250 \mathrm{mK}$; Figs. 3 and 4 , a $1 \mu \mathrm{m}$ device at $\approx 100 \mathrm{mK}$. The presented results were reproducible in other devices. For QD experiments, the emitter QPC was also used as a collector to double the electron travel length.

\section{B. Energy scaling in nonmonoenergetic TMF}

TMF acts as a spectrometer by taking advantage of the momentum dependence in cyclotron orbits [21]. An electron with charge $-e$ and momentum $p$ is deflected into its cyclotron orbit by the Lorentz force induced from an out-of-plane magnetic field $B$. The radius of said orbit is called the cyclotron radius $r_{c}=p / e B$. During TMF, a beam of electrons is emitted from a localized source, and a collector is placed at distance $L$ perpendicular to both the beam direction and magnetic field. The spreading beam is refocused at the opposite end of the cyclotron orbit, and the collection is maximized when the focal point coincides with the collector, i.e., at the focusing field $B_{f}$ :

$$
B_{f}=\frac{p}{e L / 2} .
$$

The transmission $T=I_{C} /\left(I_{C}+I_{R}\right)$ from the emitter to the collector inspected against $B$ is called the focusing spectrum. 
(a)

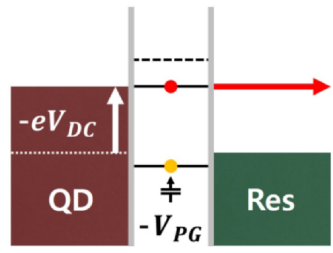

(c)

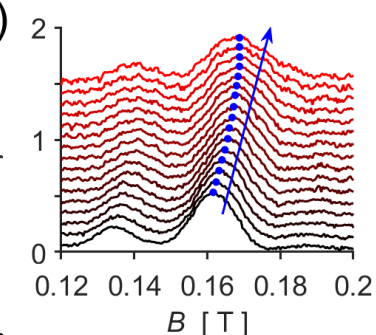

(e)

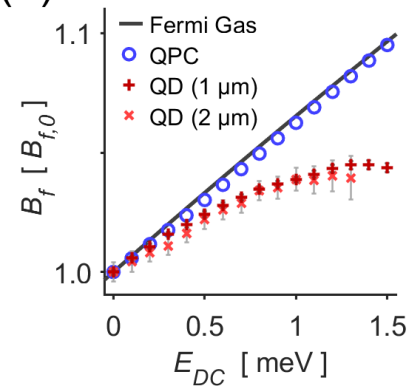

(b)

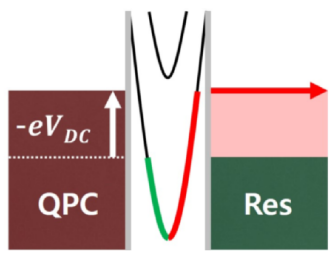

(d)

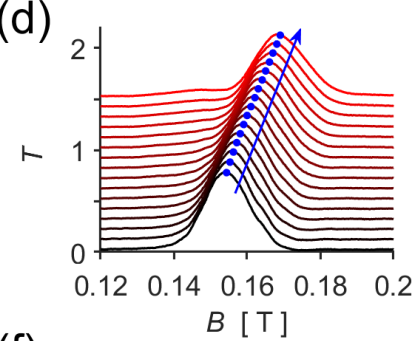

(f)

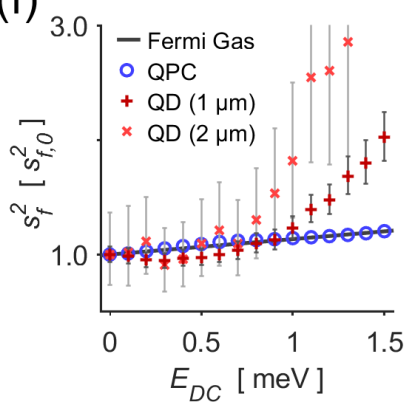

FIG. 3. Transverse magnetic focusing (TMF) spectra of quantum dot (QD)- and quantum point contact (QPC)-emitted hot electrons. (a) The QD level was aligned with the biased electrochemical potential of the lead, and (b) bidirectional, one-channel transport was maintained in the QPC. (c) The QD TMF spectra for increasing levels of $E_{\mathrm{DC}}$ (redder lines). The peak shift nonlinearity became pronounced at high $E_{\mathrm{DC}}$. (d) The QPC focusing field shifted almost linearly. The spectrum peak (e) position and (f) width obtained by fitting. The data for QD TMF for $L=2 \mu \mathrm{m}$ has also been shown. All error bars indicate a $95 \%$ confidence interval; the error bars for the QPC data were smaller than the marker and hence omitted.

The spectrum peaks at multiples of $B_{f}$ as the beam is focused first directly onto the collector then by skipping along the barrier from the emitter to the collector. Figure 1(b) is a typical example of mesoscopic TMF, measured from our sample using a QPC emitter [Fig. 1(b) inset].

The focusing spectrum scales with energy through the dispersion relation. As in Eq. (1), the spectrum peak position $B_{f}$ and width $s_{f}$ obtained at energy $E$ modulates linearly with the momentum. For Fermi gas quasiparticles, $p \propto \sqrt{E}$, and we expect the following relationship with $B_{f, 0}$ and $s_{f, 0}$ measured at $E_{0}$ :

$$
\frac{B_{f}}{B_{f, 0}}=\frac{s_{f}}{s_{f, 0}}=\sqrt{\frac{E}{E_{0}}} .
$$

From a spatial perspective, Eq. (2) simply reflects the fact that hot electrons have larger cyclotron orbits [Fig. 1(a) red curve] than their equilibrium counterparts [Fig. 1(a) yellow

curve]. The analysis can be generalized to nonmonoenergetic electron beams:

$$
\begin{aligned}
\left(\frac{B_{f}}{B_{f, 0}}\right)^{2} & \approx \frac{\epsilon}{E_{0}}, \\
\left(\frac{s_{f}}{B_{f}}\right)^{2} & \approx\left(\frac{s_{f, 0}}{B_{f, 0}}\right)^{2}+\left(\frac{\sigma}{2 \epsilon}\right)^{2},
\end{aligned}
$$

where $\epsilon$ and $\sigma^{2}$ are the mean and variance of the energy distribution. Since the focusing spectrum at equilibrium is monoenergetic, it serves as the reference with which all hot electron experiments can be compared, i.e., $E_{0}=E_{F}$. Through these results, we may use TMF to find deviations of QPC- and, more importantly, QD-emitted hot electrons from nondissipative monoenergetic excitations, i.e., Fermi gas quasiparticles. The derivation and implementation are further elaborated in Appendix A.

\section{TMF SPECTRA}

\section{A. QD-emitter TMF at equilibrium}

Performing QD-emitter TMF is nontrivial because the magnetic field affects the QD. Generally, QD levels are perturbed by the coupling between the angular momentum of the orbitals and the external magnetic field [28]. Consequently, the emitted electrons can suffer from unpredictable energy variations on the order of the QD level spacings, seen as nonmonotonic shifts of the Coulomb peak in Fig. 2(a). Such shifts are observed in the focusing spectrum as well, Fig. 2(b), and the naïve use of a QD emitter leads to undesirable changes in the hot electron energy on the order of the QD excitations. These magnetic energy shifts must be accounted for by continuous realignment of the transmitting QD level with the lead Fermi levels - constant at low magnetic fields. The appropriate adjustments to the QD level were identified by maximal conductance with respect to $V_{\mathrm{PG}}$. By tracing along the QD conductance peaks [Fig. 2(c) inset], the focusing spectrum was attained for a fixed QD level.

At equilibrium, the QD-emitted TMF was well described by the conventional analysis [20]. The first spectrum peak appears close to the semiclassical prediction, Eq. (1), and the deviation was attributed to geometric uncertainties; the lithographic gap in the QD and QPC adds up to $\approx \pm 10 \%$ of the focusing distance, leading to a similar deviation in $B_{f}$. Such imprecisions were irrelevant to our studies since Eq. (3) only requires the scaling property of the spectra. The following peaks are strongly affected by interferences between multiple paths [20,23], boundary specularity and roughness issues during skipping orbits [29], and increasing relevance of quantum Hall edge states [30]. Therefore, we restricted our interest to the first peak to avoid such misguiding effects.

\section{B. Hot electron TMF}

A clear difference was observed between QD- and QPCemitted hot electron TMF. The transmitting QD level was aligned to the biased electrochemical potential $E_{F}+E_{\mathrm{DC}}$ [Fig. 3(a)], and the QPC was tuned to retain a constant conductance $2 e^{2} / h$ [Fig. 3(b)] [31]. The focusing spectra for excitations $E_{\mathrm{DC}}=0-1.5 \mathrm{meV}$ are shown in Figs. 3(c) 
(a)

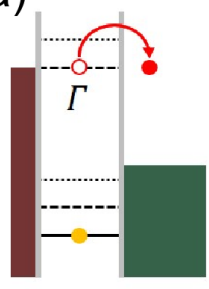

(d)

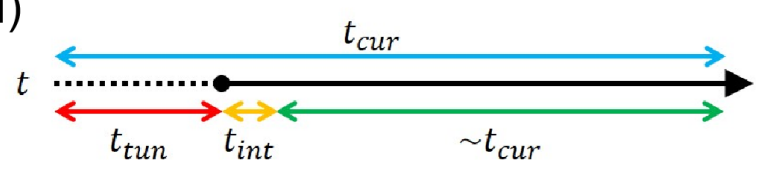

(b)

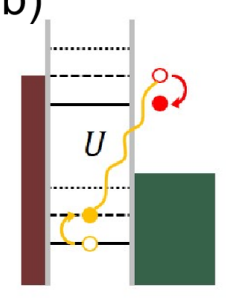

(c)

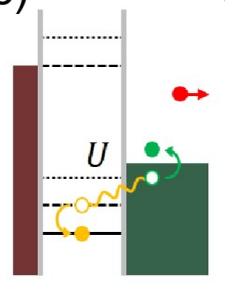

(e)

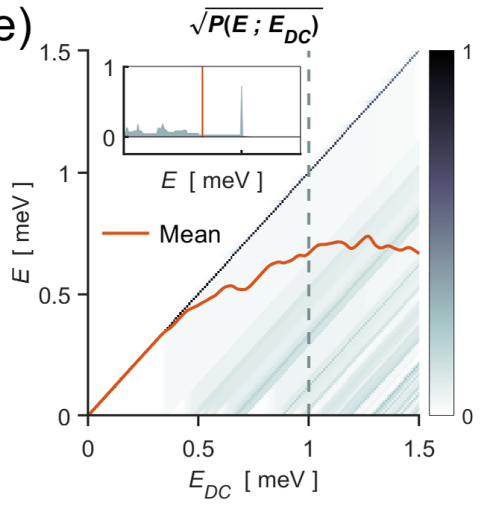

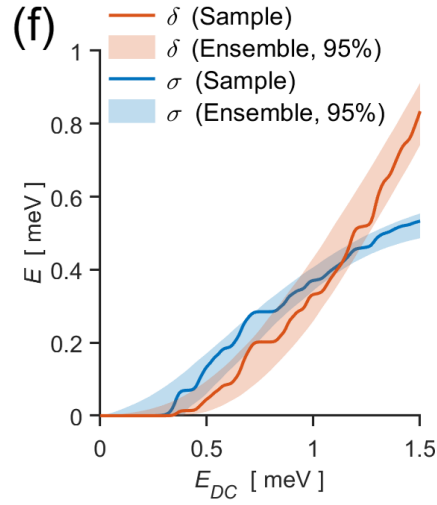

FIG. 4. Energy relaxation of quantum dot (QD)-emitted hot electrons. (a) A QD with coupling strength $\Gamma$ emits a hot electron with average periodicity $t_{\mathrm{cur}}$. The electron dwell time is on the order of $t_{\mathrm{tun}} \approx \hbar \Gamma^{-1}$. (b) The transmitted hot electron enters the lead and excites the QD through a capacitive interaction $U$ during a short time $t_{\text {int }}$. (c) For the remainder of $t_{\text {cur }}$, the QD returns to its ground state by creating electron-hole pairs through a similar capacitive interaction. (d) Among the present timescales, $t_{\text {int }}$ is the smallest. (e) Although the energy distribution of the hot electron is heavily peaked at $E_{\mathrm{DC}}$, a considerable portion is relaxed during the capacitive interaction, and the mean deviates significantly from the distribution peak. (f) The simulated mean energy loss $\delta$ and broadening $\sigma$ for a sample QD is typically within the $95 \%$ confidence interval of the ensemble.

and 3(d). For the QD-emitter experiment, the focusing peak initially shifted linear to $E_{\mathrm{DC}}$ but soon exhibited visible nonlinearities at higher biases. On the other hand, the QPC-emitter experiment presented no such behaviors. The spectrum peak positions $B_{f}$ and widths $s_{f}$ were extracted by fitting [31].

Unlike the QPC spectra, the QD spectra showed visible deviations from Fermi gas expectations. In support of previous reports that QPC hot electrons act as Fermi gas quasiparticles [21,32], we found that both the peak positions and widths of the QPC spectra scaled with $\sqrt{E_{F}+E_{\mathrm{DC}}}$, blue circles in Figs. 3(e) and 3(f). However, the QD spectra indicated a large, unexpected energy loss. The peak position $B_{f}$ from the QD spectra was considerably smaller than predicted [Fig. 3(e) red crosses], even decreasing with $E_{\mathrm{DC}}$ at some point and implying a smaller cyclotron orbit. That is, QD-emitted hot electrons propagated in the $2 \mathrm{DEG}$ with less energy than $E_{\mathrm{DC}}$. Moreover, doubling the focusing distance from $L=1$ to $2 \mu \mathrm{m}$ showed similar results [Fig. 3(e) diagonal crosses], suggesting that the hot electrons did not lose energy midflight. Considering that the mean-free path in high mobility 2DEG is typically tens of microns, our observation points to a general relaxation mechanism terminating at much shorter length scales $\ll L \approx 1 \mu \mathrm{m}$. Analysis of the peak widths provides further support that QD emissions undergo vastly different processes compared with QPC hot electrons, Fig. 3(f). The excessive broadening observed at high biases in the QD spectra points to a large redistribution of the energy of the hot electrons occurring simultaneously with the energy loss.

\section{THEORETICAL ANALYSIS AND SIMULATION}

\section{A. Relaxation model}

From the perspective of electrodynamics, QD transmission is a violent process which significantly affects nearby electrons. Therefore, we modeled the energy relaxation by introducing the capacitive interaction $H_{\text {int }}$ between the leads and the QD:

$$
H_{\mathrm{int}}=U \sum_{i, j, \mathbf{p}, \mathbf{q}} d_{i}^{\dagger} d_{j} c_{\mathbf{p}}^{\dagger} c_{\mathbf{q}},
$$

where $U$ is the energy scale for the interaction, $d_{i}^{\dagger}$ the creation operator for the $i$ th QD level, and $c_{\mathbf{p}}^{\dagger}$ the creation operator for momentum state $\mathbf{p}$ in the lead. We estimate $U \lesssim 0.8 \mathrm{meV}$ as the Coulomb potential felt by the hot electron due to the QD. The corresponding evolution of the QD was found using the master equation:

$$
\dot{P}_{\alpha}=\sum_{\beta}\left(\gamma_{\alpha \beta} P_{\beta}-\gamma_{\beta \alpha} P_{\alpha}\right),
$$

where $P_{\alpha}$ is the occupation of the $\alpha$ th QD state and $\gamma_{\beta \alpha}$ the transition rate from the $\alpha$ th to the $\beta$ th QD state given by Fermi's golden rule. The nominal rate of transitions is given by $\hbar \gamma_{0}=2 \pi U^{2} / E_{F}$. The QD excitations $\left\{\Delta_{\alpha}\right\}$ were generated by permuting orbital energies $\left\{\epsilon_{i}\right\}$, which were sampled from random variables $\left\{\hat{\epsilon}_{i}\right\}$ with mean $i \epsilon_{0}$ and variance $\epsilon_{\sigma}^{2} ; \epsilon_{0}$ denotes the average orbital level spacing, and $\epsilon_{\sigma}^{2}$ reflects the randomness in said spacings present in smaller and heavier populated QDs [33]. Although the precise estimation of $\left\{\epsilon_{i}\right\}$ is difficult, we found $\epsilon_{0}, \epsilon_{\sigma} \approx 100-200 \mu \mathrm{eV}$ for the QD used in Fig. 3. Ensembles of $1000\left\{\epsilon_{i}\right\}$ samples were analyzed for various values of $\epsilon_{0}$ and $\epsilon_{\sigma}^{2}$.

The relaxation mechanism has three stages: hot electron emission, QD excitation, and initialization. During the emission stage, Fig. 4(a), a hot electron was transmitted with an average periodicity of $t_{\text {cur }}=e /\left(I_{C}+I_{R}\right) \gtrsim 500 \mathrm{ps,} \mathrm{each} \mathrm{hav-}$ ing dwelt in the QD level for a duration on the order of $t_{\text {tun }} \approx$ 70 ps. Immediately after emission, the QD is initially in the ground state, i.e., $P_{\alpha}^{(0)}=1$ for $\alpha=0$ and 0 for all else. Then the QD is capacitively excited by the hot electron over a time $t$ from its initial state $P_{\alpha}^{(0)}$ to $P_{\alpha}^{(t)}$ via Eq. (4), Fig. 4(b). The interaction occurs within the timescale $t_{\text {int }}=l_{\text {int }} / v_{F} \lesssim 50 \mathrm{fs}$, where $v_{F}$ is the Fermi velocity, and $l_{\text {int }}$ is likely on the order of the charge screening length $\approx 10 \mathrm{~nm}$ [34]. The normalized 
interaction time $\gamma_{0} t_{\text {int }} \propto U^{2} l_{\text {int }}$ was $\approx 0.042 \ll 1$, consistent with the approximations leading to Eq. (4). After $t>t_{\text {int }}$, Fig. 4(c), the hot electron leaves the vicinity of the QD, which relaxes toward its ground state by forming electron-hole pairs in the leads through the same mechanism. Since $l_{\text {int }} \ll L$ and $L$ is much shorter than the mean-free path, no further energy relaxation is expected. Figure 4(d) summarizes the order and hierarchy of relevant timescales. Detailed discussions on the model and parameters are provided in Appendix B.

Figure 4(e) visualizes the typical energy distribution of a hot electron after exciting a QD from the ensemble $\epsilon_{0}, \epsilon_{\sigma}=$ $160 \mu \mathrm{eV}$. Invoking energy conservation, the energy distribution of a hot electron with initial energy $E_{\mathrm{DC}}$ is given by

$$
P\left(E ; E_{\mathrm{DC}}\right)=\sum P_{\alpha} \delta_{D}\left(E+\Delta_{\alpha}-E_{\mathrm{DC}}\right),
$$

where the sum is over $\alpha$ and $\delta_{D}$ is the Dirac $\delta$ function. At $E_{\mathrm{DC}} \approx 0$, the hot electron does not have enough energy to excite the QD and remains unrelaxed. At higher $E_{\mathrm{DC}}$, the energy distribution is still heavily peaked at $E_{\mathrm{DC}}$, as can be seen along $E=E_{\mathrm{DC}}$ as previous reported [35], but the amplitude leaks down to lower energies as increasingly more QD excitations become accessible. Consequently, the mean energy $\epsilon=$ $\int d E P\left(E ; E_{\mathrm{DC}}\right) E$ deviates significantly from $E_{\mathrm{DC}}$, orange line in Fig. 4(e). Although the energy distribution is small at $E<E_{\mathrm{DC}}$, the relaxation becomes significant because the relaxed distribution is spread over a considerably large range of energy [Fig. 4(e) inset]. Naturally, the distribution variance $\sigma^{2}=\int d E P\left(E ; E_{\mathrm{DC}}\right)(E-\epsilon)^{2}$ also increases with $E_{\mathrm{DC}}$. The detailed mean energy loss $\delta=E_{\mathrm{DC}}-\epsilon$ and broadening $\sigma$ depends on the precise QD levels but falls within a range reasonably predictable from the QD ensemble parameters $\epsilon_{0}$ and $\epsilon_{\sigma}^{2}$ [Fig. 4(f)] [31].

\section{B. Simulated hot electron TMF}

Our model was able to predict the key features of the QD spectra. In Figs. 5(a) and 5(b), $\delta$ and $\sigma^{2}$ extracted from experiment using Eq. (3) are juxtaposed with the ensemble values from Fig. 4(f). Both model and QD experiment presented similar qualitative tendencies with improved quantitative accuracy at higher biases. The primary effect $\delta$ was quantitatively well described by our model above $E_{\mathrm{DC}} \gtrsim$ $0.5 \mathrm{meV}$, where the relaxation effect becomes increasingly pronounced. Meanwhile, the QPC spectra presented minimal deviations from the conventional predictions, i.e., $\delta, \sigma^{2} \approx 0$. From the agreement, we believe that the interaction becomes increasingly apparent only after $E_{\mathrm{DC}}$ exceeds several QD excitation levels and opens multiple channels for the hot electron to relax.

Finally, we simulated the transmission $\langle T(B)\rangle$ from QD TMF using the focusing spectrum $T_{F}(B)$ measured at the Fermi energy. An electron beam with energy distribution $P(E)$ has the averaged spectrum $\langle T(B)\rangle=\int d E P(E) T(B ; E)$, where $T(B ; E)$ is the monoenergetic spectrum at energy $E$. Although $T(B ; E)$ is not directly obtainable, we may scale and use $T(B ; E)=T_{F}\left(B \sqrt{E_{F} / E}\right)$ since $T_{F}(B)=T\left(B ; E_{F}\right)$ is monoenergetic by virtue of equilibrium. Using our model, this
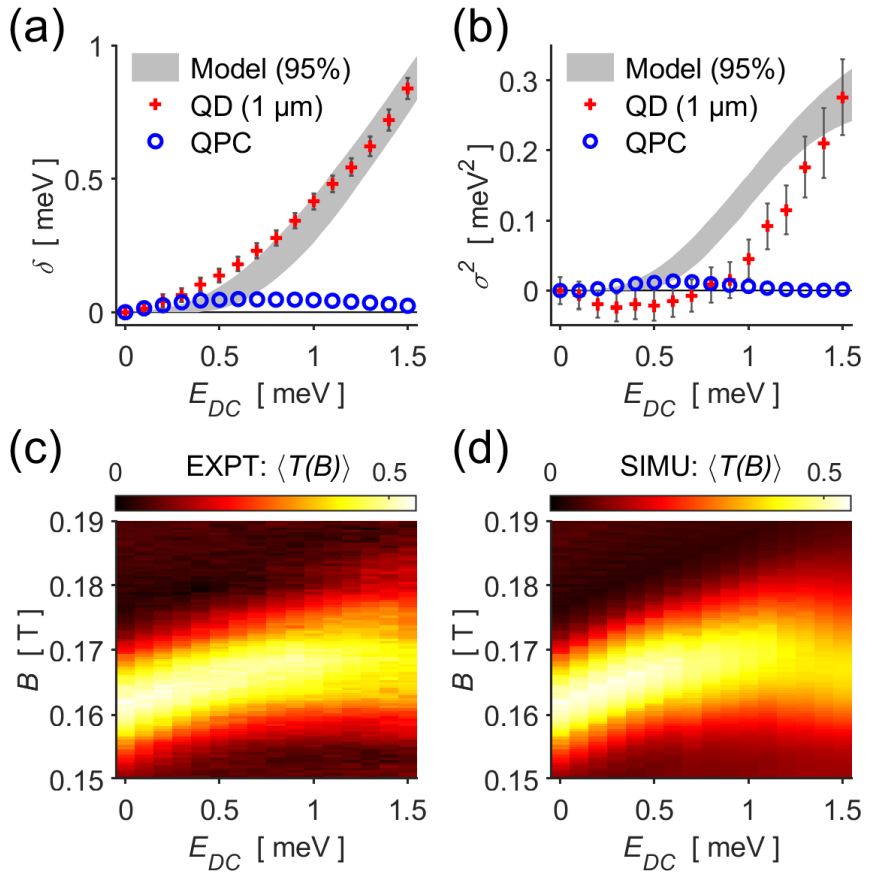

FIG. 5. Energetics of relaxation model and simulated transverse magnetic focusing (TMF) spectra. Predictions of (a) mean energy loss $\delta$ and (b) broadening $\sigma^{2}$ by the model plotted with those observed from quantum dot (QD) and quantum point contact (QPC) experiments. (c) The measured QD-emitted hot electron TMF spectra juxtaposed with (d) the nonmonoenergetic TMF spectra simulated using the model. All error bars indicate a $95 \%$ confidence interval; the error bars for the QPC data were smaller than the marker and hence omitted.

gives

$$
\langle T(B)\rangle=\sum_{\alpha} P_{\alpha} T_{F}\left(\frac{B}{\sqrt{E_{\alpha} / E_{F}}}\right),
$$

where $E_{\alpha}=\left(E_{F}+E_{\mathrm{DC}}\right)-\Delta_{\alpha}$ is the hot electron energy after exciting QD state $\alpha$. The simulated spectra for the sample shown in Fig. 4(e) highly resembles the QD spectra, both in peak position shift and width broadening [Figs. 5(c) and 5(d)] [31].

\section{DISCUSSION AND CONCLUSIONS}

Although our relaxation mechanism originates from a generic capacitive interaction, we expect it to be inconsequential during QPC emissions for two major reasons. Firstly, QPC emissions experience a much weaker interaction with the 2D reservoir than QD emissions. Since no depletion region exists between the QPC and the 2D reservoir, electrostatic interactions between the two are highly screened. Also, QPC emission is a continuous process and does not incur the sudden and violent charge rearrangement present during QD emission. Furthermore, QPCs emit far more electrons than a QD does at the high biases where relaxation became apparent. Therefore, the effective interaction-and consequently the relaxation rate-between the QPC-emitted hot electron in the $2 \mathrm{D}$ reservoir and the leftover QPC electrons is expected to be much smaller. Secondly, the relaxation of QPC hot electrons is 
suppressed by the fermionic nature of electrons. A QPC transmits at the biased Fermi level as well as all energies spanning down to the grounded Fermi level. The emitted electrons are localized as a collimated ray-as can be seen in the nearly unitary transmission at the focusing peak-so only a small fraction of the 2D density of states may participate as potential relaxation channels. Consequently, QPC emissions with energy below the highest occupied level prevent the relaxation of QPC hot electrons via Pauli exclusion with effectiveness proportional to the collimation. These reasons suggest a few directions for further research. For example, a detailed study of the QD-reservoir coupling dependence would provide a means to investigate the importance of capacitive interactions and the transition from QPC-like to QD-like emissions. Also, the use of QPC emitters at the unidirectional configuration may illuminate the role of collimation in relaxation suppression.

In summary, the energetic properties of hot electrons were analyzed via TMF with QD and QPC emitters. The measured focusing spectra revealed an energy relaxation during QD emission on the order of the initial hot electron energy, while the QPC spectra closely followed Fermi gas predictions. The relaxation was modeled by considering a capacitive interaction between the QD and the leads. The model predicted that the hot electron loses energy by exciting the QD emitter; the energy loss and broadening from the model accounted for experimental observations with increasing accuracy over $E_{\mathrm{DC}}>0.5 \mathrm{meV}$, where the relaxation became more apparent. The simulated TMF spectra based on the model showed close resemblance with the observed QD spectra. Our results suggest that QDs may not be reliable hot electron sources for energies higher than the QD excitations, even if the excited levels do not directly participate in electron transmission. We believe that our analysis supports the presence of a large, unexpected relaxation during QD transport and will prove crucial in further studies of quasiparticle relaxations, especially near local potential traps or impurities.

\section{ACKNOWLEDGMENTS}

We thank M. Heiblum for his experimental support (access to his facilities) and D. Mahalu for her help in nanofabrication. We also thank H. C. Park, N. Myoung, and S.-J. Choi for their discussions. This paper was supported by a National Research Foundation of Korea (NRF) grant funded by the Korean Government (MSIP; NRF-2016R1A5A1008184), and H.K.C. was partially supported by NRF-2017R1C1B3004301.

H.C. and H.K.C. jointly supervised this paper.

\section{APPENDIX A: TMF SPECTRUM LINESHAPE ANALYSIS}

A classical free electron with momentum $p$ has a cyclotron radius $r_{c}=p / e B$, where $-e$ is the electron charge, and $B$ is the magnetic field strength. In the TMF geometry, a collimated beam of electrons leaves the emitter with some angular distribution $C(\theta)$. Due to the magnetic field, an electron leaving with angle $\theta$ is deflected back to $x=2 r_{c} \cos (\theta)$ on the barrier. The distribution $g_{0}^{\operatorname{sign}(\theta)}(x)$ of electrons at the barrier is given by

$$
g_{0}^{ \pm}(x)=\frac{1}{2 r_{c}} \frac{C\left[ \pm \operatorname{acos}\left(x / 2 r_{c}\right)\right]}{\sqrt{1-\left(x / 2 r_{c}\right)^{2}}} .
$$

The electrons are then reflected by the barrier and enter an extended trajectory called skipping orbits. The electron distribution after $n$ reflections is given by $g_{n}^{ \pm}(x)=$ $g_{0}^{ \pm}\left[r_{c} \rightarrow(n+1) r_{c}\right]$. The divergences at $x=2(n+1) r_{c}$ are due to the formation of caustics, which coincides with where center electrons, i.e., $\theta=0$, arrive. Hence, electrons are said to be focused onto $x=2(n+1) r_{c}$.

A collector of width $l$ at $x=L$ on the barrier drains the impinging electrons. The ratio between collected and total electrons is the transmission $T=\int d \theta C(\theta)$, where the integral is done over the angular intervals of collected electrons. The transmission as a function of $B$ is called the TMF spectrum and in the limit $l \rightarrow 0$ is given by

$$
\frac{T(B)}{l / L} \rightarrow \sum_{ \pm, n} \frac{1}{n+1} \frac{C\left\{ \pm \operatorname{acos}\left[B /(n+1) B_{0}\right]\right\}}{\sqrt{1-\left[B /(n+1) B_{0}\right]^{2}}},
$$

where $B_{0}=2 p / e L$ is the focusing field, and the summation is done over $B / B_{0}-1 \leqslant n$. The divergences at $B=(n+1) B_{0}$ indicate electrons being directly focused onto the collector; in practice, the spectrum has finite peaks due to the finite width of the emitter and collector. Henceforth, we will focus on the principal peak at $n=0$.

The spectrum scales linearly with $B_{0}$. Since the energy $E$ of free electrons is quadratic in $p, B_{0} \propto p \propto \sqrt{E}$, and we expected the focusing field $B_{0}$ to scale from a reference energy $E_{0}$ as

$$
\begin{aligned}
& E_{0} \rightarrow E, \\
& B_{0} \rightarrow B_{0} \sqrt{\frac{E}{E_{0}}} .
\end{aligned}
$$

Therefore, we can express the scaling property of the spectrum $T(B \mid E)$ measured at $E$ as

$$
\begin{aligned}
& E_{0} \rightarrow E, \\
& T\left(B \mid E_{0}\right) \rightarrow T(B \mid E)=T\left(B \sqrt{\frac{E_{0}}{E}} \mid E_{0}\right) .
\end{aligned}
$$

Such scaling is also present in semiclassical solutions of the TMF spectrum [23], and we assume the property to be inherited by mesoscopic TMF as well. In extension, the monoenergetic spectrum $T(B \mid E)$ can be generalized to a spectrum $\langle T(B \mid E)\rangle$ incoherently summed over some probability distribution $P(E)$ :

$$
\langle T(B \mid E)\rangle=\sum_{E} P(E) T\left(B \sqrt{\frac{E_{0}}{E}} \mid E_{0}\right) .
$$

With a few assumptions, we can relate the peak statistics of a mildly energy-broadened spectrum to those of a monoenergetic one. Let the distribution $P(E)$ be heavily centered about its mean $\epsilon=\sum P(E) E$ with variance $\sigma^{2}=\sum P(E)(E-\epsilon)^{2}$. To the leading order, we expect the peak position $\left\langle B_{0}\right\rangle$ of the energy-broadened spectrum $\langle T\rangle$ to be at

$$
\left\langle B_{0}\right\rangle / B_{0}=\sum_{E} P(E) \sqrt{\frac{E}{E_{0}}} \approx \sqrt{\frac{\epsilon}{E_{0}}} .
$$


Now we define the peak width $s_{0}$ as the standard deviation of the monoenergetic spectrum $T(B)$, with $E$ suppressed about $B_{0}$, i.e.,

$$
s_{0}^{2}=\int d B T_{N}(B)\left(B-B_{0}\right)^{2},
$$

where $T_{N}(B)=T(B) / \int d B T(B)$. Note that it satisfies the same scaling properties as $B_{0}$. The width quantifies the amount of geometric broadening present in TMF, chiefly determined by the collimation of the beam and the regime in which the experiment is done. Similarly, the width $\left\langle s_{0}\right\rangle$ of spectrum $\langle T\rangle$ is defined as

$$
\left\langle s_{0}\right\rangle^{2}=\int d B\langle T(B)\rangle_{n}\left(B-\left\langle B_{0}\right\rangle\right)^{2},
$$

where $\langle T(B)\rangle_{N}=\langle T(B)\rangle / \int d B\langle T(B)\rangle$. For convenience, we introduce an energy-weighted distribution $Q(E)$ :

$$
Q(E)=\frac{P(E) \sqrt{E}}{\sum P\left(E^{\prime}\right) \sqrt{E^{\prime}}} .
$$

Then Eq. (A7) can be rewritten as

$$
\left\langle s_{0}\right\rangle^{2} \approx \sum_{E} Q(E) \frac{E}{E_{0}} \int d B T_{N}(B)\left(B-\left\langle B_{0}\right\rangle \sqrt{\frac{E_{0}}{E}}\right)^{2} .
$$

To the leading orders in $s_{0}$ and $\sigma$, this reduces to

$$
\begin{aligned}
\left\langle s_{0}\right\rangle^{2} & \approx\left[\frac{s_{0}^{2}}{B_{0}^{2}}+(1+\xi) \frac{1}{4} \frac{\sigma^{2}}{\epsilon^{2}}\right]\left\langle B_{0}\right\rangle^{2}, \\
\xi & :=3\left(\frac{\bar{B}}{B_{0}}-1\right),
\end{aligned}
$$

where $\bar{B}=\int d B T_{N}(B) B$, and $\xi$ is a variable quantifying nonidealness of $B_{0}$ as an estimator of $\bar{B}$. For a monoenergetic spectrum sufficiently sharp and symmetric about $B_{0}$, i.e., $\xi \ll 1$, the leading terms simplify to

$$
\left(\frac{1}{2} \frac{\sigma}{\epsilon}\right)^{2} \approx\left(\frac{\left\langle s_{0}\right\rangle}{\left\langle B_{0}\right\rangle}\right)^{2}-\left(\frac{s_{0}}{B_{0}}\right)^{2}
$$

The results were implemented in our experiment analysis as follows. The mean energy $\epsilon$ is given by

$$
\epsilon=E_{F}+E_{\mathrm{DC}}-\delta,
$$

where $E_{\mathrm{DC}}$ is the energy initially supplied to the hot electron in excess of $E_{F}$, and $\delta$ is the mean energy relaxation. Since no relaxation is present at the Fermi level, we recognize the spectrum at $E_{0}=E_{F}$ to be monoenergetic. Then the peak positions $B_{f}$ and widths $s_{f}$ from the experimental spectra measured are

$$
\begin{gathered}
B_{f}=\left\{\begin{array}{cc}
B_{0} & E_{\mathrm{DC}}=0 \\
\left\langle B_{0}\right\rangle & E_{\mathrm{DC}} \neq 0,
\end{array}\right. \\
s_{f}=\left\{\begin{array}{cc}
s_{0} & E_{\mathrm{DC}}=0 \\
\left\langle s_{0}\right\rangle & E_{\mathrm{DC}} \neq 0 .
\end{array}\right.
\end{gathered}
$$

In the main text, we specified $B_{f}\left(E_{\mathrm{DC}}=0\right)=B_{f, 0}$ and $s_{f}\left(E_{\mathrm{DC}}=0\right)=s_{f, 0}$. In practice, $B_{f}$ and $s_{f}$ were found by Gaussian fitting the spectra; the Gaussian function provided the best fit among other candidate functions. Our main results,
Eqs. (A5) and (A11), can be rewritten as

$$
\begin{aligned}
\frac{\delta}{E_{F}} & =\left(1+\frac{E_{\mathrm{DC}}}{E_{F}}\right)-\left(\frac{B_{f}}{B_{f, 0}}\right)^{2}, \\
\left(\frac{\sigma}{E_{F}}\right)^{2} & =4\left(\frac{B_{f}}{B_{f, 0}}\right)^{4}\left[\left(\frac{s_{f}}{B_{f}}\right)^{2}-\left(\frac{s_{f, 0}}{B_{f, 0}}\right)^{2}\right],
\end{aligned}
$$

where we have used $\sqrt{\epsilon / E_{F}}=B_{f} / B_{f, 0}$. This form was used for Fig. 4(f) in the main text. Conversely, we may also write

$$
\begin{aligned}
\frac{B_{f}}{B_{f, 0}} & =\sqrt{\left(1+\frac{E_{\mathrm{DC}}-\delta}{E_{F}}\right)} \\
\left(\frac{s_{f}}{s_{f, 0}}\right)^{2} & =\left(\frac{\epsilon}{E_{F}}\right)\left[1+\left(\frac{\sigma}{2 \epsilon}\right)^{2} /\left(\frac{s_{f, 0}}{B_{f, 0}}\right)^{2}\right],
\end{aligned}
$$

which we have used in the fitting benchmark [31].

\section{APPENDIX B: RELAXATION MODEL AND PARAMETER ESTIMATION}

The model starts with the well-understood spinless QDlead tunneling system described by Hamiltonian $H_{0}$ [36]:

$$
\begin{aligned}
H_{0} & =H_{\mathrm{QD}}+H_{\text {lead }}+H_{\mathrm{tun}}, \\
H_{\mathrm{QD}} & =\sum_{i} \epsilon_{i} n_{i}+\epsilon_{c} \sum_{i<j} n_{i} n_{j}, \\
H_{\text {lead }} & =\sum_{\mathbf{p}, \lambda} \epsilon_{\mathbf{p}, \lambda} n_{\mathbf{p}, \lambda}, \\
H_{\text {tun }} & =\sum_{i, \lambda} \Gamma_{i, \lambda} d_{i}^{\dagger} \psi_{\lambda}(x=0)+\text { H.c. }
\end{aligned}
$$

where $d_{i}^{\dagger}$ is the creation operator for the $i$ th QD orbital, $n_{i}=$ $d_{i}^{\dagger} d_{i}$ the corresponding number operator, $c_{\mathbf{p}, \lambda}^{\dagger}$ the creation operator for the momentum state $\mathbf{p}$ in lead $\lambda, n_{\mathbf{p}, \lambda}=c_{\mathbf{p}, \lambda}^{\dagger} c_{\mathbf{p}, \lambda}$ the corresponding number operator, $\psi_{\lambda}(x=0)=\frac{1}{A} \sum_{\mathbf{p}} c_{\mathbf{p}, \lambda}$ with normalization constant $A$, and H.c. the Hermitian conjugate of the preceding term. Here, $H_{\mathrm{QD}}$ counts the energy of occupied orbitals $\Sigma_{i} \epsilon_{i} n_{i}$ and the capacitive charging energy between occupied orbitals $\epsilon_{c} \Sigma_{i<j} n_{i} n_{j} ; H_{\text {lead }}$ is the usual Fermi gas Hamiltonian, where $\epsilon_{\mathbf{p}, \lambda}=\mathbf{p}^{2} / 2 m^{*}$; and $H_{\text {tun }}$ creates the hopping $\Gamma_{i, \lambda}$ from the lead $\psi_{\lambda}$ to the orbital $d_{i}^{\dagger}$ in the vicinity of the QD, and vice versa. The many-body state $\phi$ of the QD is given by $\Pi_{i \in \phi} d_{i}^{\dagger}$ and is indexed by the energetic order $\alpha$; the excitations $\Delta_{\alpha}$ are defined with respect to the ground state $\alpha=0$, i.e., $\Delta_{0}=0$ and $\Delta_{\alpha} \leqslant \Delta_{\alpha+1}$. The total state is then written as $|\alpha \mathbf{p}\rangle$ to describe the total state in which the QD is in state $\phi_{\alpha}$, and a hot electron is in one of the leads with momentum $\mathbf{p}$ over the Fermi sea.

We introduced a capacitive interaction to relax the hot electron in the lead by exciting the QD levels, i.e., the total system is described by $H$ :

$$
\begin{aligned}
H & =H_{0}+H_{\text {int }}, \\
H_{\text {int }} & =\bar{U} \sum_{i, j, \lambda} d_{i}^{\dagger} d_{j} \rho_{\lambda}(x=0) .
\end{aligned}
$$

where $\quad \rho_{\lambda}(x=0)=\psi_{\lambda}^{\dagger}(x=0) \psi_{\lambda}(x=0), \quad$ and $\quad \bar{U}$ parametrizes the Coulomb force between the QD and the 
charge in the leads. Note that the interaction is defined to be short ranged in $\rho_{\lambda}(x=0)$. Expanding the density operator and rewriting $U=\bar{U} / A^{2}$, Eq. (B2b) becomes

$$
H_{\mathrm{int}}=U \sum_{i, j, \mathbf{p}, \mathbf{q}, \lambda} d_{j}^{\dagger} d_{i} c_{\mathbf{q}, \lambda}^{\dagger} c_{\mathbf{p}, \lambda} .
$$

Through $H_{\text {int }}$, an electron in the occupied QD orbital $i$ can hop to an unoccupied orbital $j$ by scattering the hot electron in lead $\lambda$ from momentum $\mathbf{p}$ to $\mathbf{q}$, i.e., $|\alpha \mathbf{p}\rangle \rightarrow d_{j}^{\dagger} d_{i} c_{\mathbf{q}}^{\dagger} c_{\mathbf{p}}|\alpha \mathbf{p}\rangle$. Fermi's golden rule gives us the transition rate $\gamma_{\beta \alpha}$ from QD state $\alpha$ to $\beta$ due to all possible scatterings from $\mathbf{p}$ to $\mathbf{q}$ :

$$
\gamma_{\alpha \beta}=\frac{2 \pi}{\hbar} \sum_{\mathbf{p}, \mathbf{q}}\left|\left\langle\beta \mathbf{q}\left|H_{\text {int }}\right| \alpha \mathbf{p}\right\rangle\right|^{2} W_{\alpha \mathbf{p}} \delta\left(E_{\alpha \mathbf{p}}-E_{\beta \mathbf{q}}\right),
$$

where $W_{\alpha \mathbf{p}}$ is the thermal weighting for $|\alpha \mathbf{p}\rangle$, and $E_{\alpha \mathbf{p}}=\Delta_{\alpha}+$ $\epsilon_{\mathbf{p}}$ is the total excitation from the ground state. By identifying $E_{\alpha \mathbf{p}}=E_{\beta \mathbf{q}}=E_{\mathrm{DC}}$, Eq. (B3) reduces to

$$
\begin{aligned}
& \gamma_{\beta \alpha}=\gamma_{0}\left[1-f\left(E_{\mathrm{DC}}-\Delta_{\alpha}\right)\right]\left[1-f\left(E_{\mathrm{DC}}-\Delta_{\beta}\right)\right], \\
& \hbar \gamma_{0}=2 \pi \frac{U^{2}}{E_{F}},
\end{aligned}
$$

where $f(E)=1 /\left[1+\exp \left(E / k_{B} T_{e}\right)\right]$ is the Fermi-Dirac distribution for an electron temperature $T_{e}$. Note that $\gamma_{0}$ characterizes the rate of allowed transitions $E_{\mathrm{DC}}>\Delta_{\alpha, \beta}$ at zero temperature. The initializing process for the excited QD can be solved in a similar manner with the approximation that $\Delta_{\alpha} \gg k_{B} T_{e}$, resulting in a transition rate $\gamma_{\beta \alpha}^{\prime}$ :

$$
\gamma_{\beta \alpha}^{\prime}=\gamma_{0} \frac{\left|\Delta_{\alpha}-\Delta_{\beta}\right|}{E_{F}} \Theta\left(\Delta_{\alpha}-\Delta_{\beta}\right),
$$

where $\Theta$ is the Heaviside step function. This process describes how the QD excitation is relaxed by creating electron-hole pairs in the leads. The rate is doubled if we consider the QD to be relaxing through both leads.

The master equation was used to solve the changes in $\mathrm{QD}$ state:

$$
\dot{P}_{\alpha}=\sum_{\beta}\left(\gamma_{\alpha \beta} P_{\beta}-\gamma_{\beta \alpha} P_{\alpha}\right),
$$

where $P_{\alpha}$ is the probability that the QD is in state $\phi_{\alpha}$. The initial condition is given by $P_{\alpha=0}^{(0)}=1$ and $P_{\alpha \neq 0}^{(0)}=0$, i.e., the QD starts in its ground state. After evolving the probabilities over the interaction duration between the QD and the hot electron, we are left with the distribution $P_{\alpha}^{(\tau)}$ from which we can derive the excitation mean $\delta$ and variance $\sigma^{2}$ of the QD:

$$
\begin{aligned}
\delta & =\sum_{\alpha} P_{\alpha}^{(\tau)} \Delta_{\alpha}, \\
\sigma^{2} & =\sum_{\alpha} P_{\alpha}^{(\tau)}\left(\Delta_{\alpha}-\delta\right)^{2} .
\end{aligned}
$$

After the hot electron leaves the vicinity of the QD, the QD is left to relax through a similar process back toward its initial state $P_{\alpha} \rightarrow P_{\alpha}^{(0)}$ at the rate given in Eq. (B5). Although $\gamma_{\beta \alpha}^{\prime}$ is smaller than $\gamma_{\beta \alpha}$ by a factor of $\left|\Delta_{\alpha}-\Delta_{\beta}\right| / E_{F}$, the difference is often compensated for by the large difference in durations of each process.

Our model for hot electron energy relaxation has two types of constants: process time scales and interaction strengths. Let $t_{\text {cur }}, t_{\text {tun }}$, and $t_{\text {int }}$ be the mean period of electron transmission through the QD, the lifetime of the electron in the transmitting QD state, and the duration of interaction between the hot electron and the QD, respectively. The linear response current $I$ from the left to the right of the QD is given by

$$
I=\frac{e}{h} \int d E \frac{\Gamma_{L} \Gamma_{R}}{\left(E-E_{0}\right)^{2}+(\Gamma / 2)^{2}}\left[f\left(E-\mu_{L}\right)-f_{R}\left(E-\mu_{R}\right)\right],
$$

where $\Gamma_{\lambda}$ is the QD orbital lifetime broadening due to the coupling with lead $\lambda, \Gamma=\Gamma_{L}+\Gamma_{R}$, and $\mu_{\lambda}$ the lead electrochemical potentials. For the QD level used in the experiments shown in Figs. 3 and 4 , we estimate $I \approx 277 \mathrm{pA}$ and $\Gamma=$ $9.33 \mu \mathrm{eV}$ (c.f. Fig. S1 in the Supplemental Material [31]); I was estimated by integrating the measured QD conductance up to the Coulomb peak center since the QD level was set to the biased electrochemical potential. This gives us the time scales $t_{\text {cur }}=e / I \approx 578 \mathrm{ps}$ and $t_{\text {tun }}=\hbar / \Gamma \approx 70.4 \mathrm{ps}$. The interaction time scale $t_{\text {int }}$ is upper-bounded by the time taken for the slowest electron to reach the collector; the slowest electron is that at the Fermi level with velocity $v_{F} \approx 1.97 \times$ $10^{5} \mathrm{~ms}$, and the orbit length is $\pi L / 2=1.57 \mu \mathrm{m}$, giving us $t_{\text {int }}<8$ ps - clearly the smallest of the presented time scales. In numerical simulations, we used a much smaller estimate $t_{\text {int }}=l_{\text {int }} / v_{F} \approx 51 \mathrm{fs}$, where $l_{\text {int }} \approx 10 \mathrm{~nm}$ is the interaction length scale interpreted as the dielectric screening length in GaAs. The QD initialization takes place during the time left over, i.e., $t_{\text {cur }}-\left(t_{\text {tun }}+t_{\text {int }}\right) \sim t_{\text {cur }}$, which is at least upward of $500 \mathrm{ps} / 8 \mathrm{ps} \approx 60$ and likely on the order of $500 \mathrm{ps} / 50 \mathrm{fs}=$ 10000 times $t_{\text {int }}$.

The parameters of the model are chiefly comprised of energy scales involved. The QD is parametrized by its set of orbital levels $\left\{\epsilon_{i}\right\}$, which are combined to form the excitations $\Delta_{\alpha}$. Due to the randomness from chaotic motion in QDs, $\left\{\epsilon_{i}\right\}$ is randomly distributed with a mean interval $\epsilon_{0}$ and variance $\epsilon_{\sigma}^{2}$, i.e., $\epsilon_{i} \sim \hat{\epsilon}_{i}$, where $\hat{\epsilon}_{i}$ is a distribution with mean $i \epsilon_{0}$ and variance $\epsilon_{\sigma}^{2}$. We estimate $\epsilon_{0}$ to be in the range of 100-200 $\mu \mathrm{eV}$ and $\epsilon_{\sigma} \gtrsim \epsilon_{0}$ (c.f. Fig. S6 in the Supplemental Material [31]). Since QD transitions allow only one orbital occupation to be exchanged with another, $\left|\Delta_{\alpha}-\Delta_{\beta}\right|=\left|\epsilon_{i}-\epsilon_{j}\right|$, where the $i$, $j$ th orbitals are switched during the $\alpha \leftrightarrow \beta$ transition. Since the expected value of $\left|\epsilon_{i}-\epsilon_{j}\right|$ is $|i-j| \epsilon_{0}$, we can estimate $\left|\Delta_{\alpha}-\Delta_{\beta}\right| \gtrsim \epsilon_{0} \approx 150 \mu \mathrm{eV}$. The interaction strength $U$ is estimated by the Coulomb potential felt by the hot electron immediately across the depleted GaAs region from the QD. The QD has a net charge $|q|<e$, and the depleted region is $\approx 150 \mathrm{~nm}$ in width, giving us $\lesssim 0.8 \mathrm{meV}$; from Eq. (B4b), $\gamma_{0} \approx 823 \mathrm{THz}$.

The energy estimates allow us to parametrize the duration of each stage of the process. We define the normalized interaction time $\tau:=\gamma_{0} t_{\text {int }}$, which describes how much the interaction has progressed; note that $\tau \propto U^{2} l_{\text {int }}$. Since $\tau \approx$ $0.042 \ll 1$ from our estimate, our use of Fermi's golden rule and the master equation is justified. The QD initialization is parametrized by $\tau^{\prime} \sim \gamma_{\beta \alpha}^{\prime} t_{\text {cur }}$. Approximately, $\tau^{\prime} \gtrsim$ $\left(\epsilon_{0} / E_{F}\right)\left(\tau_{\text {cur }} / \tau_{\text {int }}\right) \tau$, which is lower bounded by $\tau^{\prime} \gg 0.25$ using $l_{\text {int }} \leqslant 1.51 \mu \mathrm{m}$ and likely on the order of $\tau^{\prime} \gtrsim 42 \gg 1$ using $l_{\text {int }} \approx 10 \mathrm{~nm}$, supporting the expectation that the QD is indeed initialized to its ground state before a new hot electron is emitted. 
[1] A. Sommerfeld, Zur elektronentheorie der metalle auf grund der fermischen statistik, Z. Phys. 47, 1 (1928).

[2] J. Bardeen, L. N. Cooper, and J. R. Schrieffer, Theory of superconductivity, Phys. Rev. 108, 1175 (1957).

[3] R. B. Laughlin, Quantized Hall conductivity in two dimensions, Phys. Rev. B 23, 5632 (1981).

[4] P. Wölfle, Quasiparticles in condensed matter systems, Rep. Prog. Phys. 81, 032501 (2018).

[5] M. L. Cohen and S. G. Louie, Fundamentals of Condensed Matter Physics (Cambridge University Press, Cambridge, 2016).

[6] M. Yamamoto, S. Takada, C. Bäuerle, K. Watanabe, A. D. Wieck, and S. Tarucha, Electrical control of a solid-state flying qubit, Nat. Nanotechnol. 7, 247 (2012).

[7] G. Fève, A. Mahé, J.-M. Berroir, T. Kontos, B. Plaçais, D. C. Glattli, A. Cavanna, B. Etienne, and Y. Jin, An on-demand coherent single-electron source, Science 316, 1169 (2007).

[8] C. Bäuerle, D. Christian Glattli, T. Meunier, F. Portier, P. Roche, P. Roulleau, S. Takada, and X. Waintal, Coherent control of single electrons: a review of current progress, Rep. Prog. Phys. 81, 056503 (2018).

[9] D. Pines, A collective description of electron interactions: IV. Electron interaction in metals, Phys. Rev. 92, 626 (1953).

[10] G. F. Giuliani and J. J. Quinn, Lifetime of a quasiparticle in a two-dimensional electron gas, Phys. Rev. B 26, 4421 (1982).

[11] I. P. Levkivskyi and E. V. Sukhorukov, Energy relaxation at quantum Hall edge, Phys. Rev. B 85, 075309 (2012).

[12] D. Ferraro, B. Roussel, C. Cabart, E. Thibierge, G. Fève, Ch. Grenier, and P. Degiovanni, Real-Time Decoherence of Landau and Levitov Quasiparticles in Quantum Hall Edge Channels, Phys. Rev. Lett. 113, 166403 (2014).

[13] U. Sivan, M. Heiblum, and C. P. Umbach, Hot Ballistic Transport and Phonon Emission in a Two-Dimensional Electron Gas, Phys. Rev. Lett. 63, 992 (1989).

[14] C. Altimiras, H. le Sueur, U. Gennser, A. Cavanna, D. Mailly, and F. Pierre, Tuning Energy Relaxation along Quantum Hall Channels, Phys. Rev. Lett. 105, 226804 (2010).

[15] Q. Weng, S. Komiyama, L. Yang, Z. An, P. Chen, S. A. Biehs, Y. Kajihara, and W. Lu, Imaging of nonlocal hot-electron energy dissipation via shot noise, Science 360, 775 (2018).

[16] T. Krähenmann, S. G. Fischer, M. Röösli, T. Ihn, C. Reichl, W. Wegscheider, K. Ensslin, Y. Gefen, and Y. Meir, Augerspectroscopy in quantum Hall edge channels and the missing energy problem, Nat. Commun. 10, 3915 (2019).

[17] R. H. Rodriguez, F. D. Parmentier, D. Ferraro, P. Roulleau, U. Gennser, A. Cavanna, M. Sassetti, F. Portier, D. Mailly, and P. Roche, Relaxation and revival of quasiparticles injected in an interacting quantum Hall liquid, Nat. Commun. 11, 2426 (2020)

[18] C. Altimiras, H. le Sueur, U. Gennser, A. Cavanna, D. Mailly, and F. Pierre, Non-equilibrium edge-channel spectroscopy in the integer quantum Hall regime, Nat. Phys. 6, 34 (2009).

[19] H. le Sueur, C. Altimiras, U. Gennser, A. Cavanna, D. Mailly, and F. Pierre, Energy Relaxation in the Integer Quantum Hall Regime, Phys. Rev. Lett. 105, 056803 (2010).

[20] H. van Houten, C. W. J. Beenakker, J. G. Williamson, M. E.
I. Broekaart, P. H. M. van Loosdrecht, B. J. van Wees, J. E. Mooij, C. T. Foxon, and J. J. Harris, Coherent electron focusing with quantum point contacts in a two-dimensional electron gas, Phys. Rev. B 39, 8556 (1989).

[21] J. G. Williamson, H. van Houten, C. W. J. Beenakker, M. E. I. Broekaart, L. I. A. Spendeler, B. J. van Wees, and C. T. Foxon, Hot-electron spectrometry with quantum point contacts, Phys. Rev. B 41, 1207 (1990).

[22] V. S. Tsoi, J. Bass, and P. Wyder, Studying conductionelectron/interface interactions using transverse electron focusing, Rev. Mod. Phys. 71, 1641 (1999).

[23] S. Bladwell and O. P. Sushkov, Interference effects and Huygens principle in transverse magnetic focusing of electrons and holes, Phys. Rev. B 96, 035413 (2017).

[24] T. M. Chen, M. Pepper, I. Farrer, G. A. C. Jones, and D. A. Ritchie, All-Electrical Injection and Detection of a SpinPolarized Current Using 1D Conductors, Phys. Rev. Lett. 109, 177202 (2012).

[25] Y. J. Um, Y. H. Oh, M. Seo, S. Lee, Y. Chung, N. Kim, V. Umansky, and D. Mahalu, Quantum point contact with large subband energy spacings, Appl. Phys. Lett. 100, 183502 (2012).

[26] D. T. Park, S. Lee, U. Kim, H. Choi, and H. K. Choi, Robust quantum point contact via trench gate modulation, Sci. Rep. 10, 19746 (2020).

[27] A. V. Kretinin and Y. Chung, Wide-band current preamplifier for conductance measurements with large input capacitance, Rev. Sci. Instrum. 83, 084704 (2012).

[28] L. P. Kouwenhoven, D. G. Austing, and S. Tarucha, Fewelectron quantum dots, Rep. Prog. Phys. 64, 701 (2001).

[29] K. Tsukagoshi, S. Takaoka, K. Murase, K. Gamo, and S. Namba, Investigation of ballistic elastic scattering length and specularity in multiterminal GaAs/AlGaAs by magnetic electron focusing effect, Appl. Phys. Lett. 62, 1609 (1993).

[30] T. Stegmann, D. E. Wolf, and A. Lorke, Magnetotransport along a boundary: from coherent electron focusing to edge channel transport, New J. Phys. 15, 113047 (2013).

[31] See Supplemental Material at http://link.aps.org/supplemental/ 10.1103/PhysRevResearch.3.033015 for details on device characterization, data processing, simulation, and fitting benchmark.

[32] T. M. Chen, M. Pepper, I. Farrer, D. A. Ritchie, and G. A. C. Jones, Magnetic focusing with quantum point contacts in the non-equilibrium transport regime, Appl. Phys. Lett. 103, 093503 (2013).

[33] C. W. J. Beenakker, Random-matrix theory of quantum transport, Rev. Mod. Phys. 69, 731 (1997).

[34] S. Datta, Electronic Transport in Mesoscopic Physics (Cambridge University Press, Cambridge, 1997).

[35] C. Rössler, S. Burkhard, T. Krähenmann, M. Röösli, P. Märki, J. Basset, T. Ihn, K. Ensslin, C. Reichl, and W. Wegscheider, Spectroscopy of equilibrium and nonequilibrium charge transfer in semiconductor quantum structures, Phys. Rev. B 90, 081302(R) (2014).

[36] H. Bruus and K. Flensberg, Transport in Interacting Mesoscopic Systems, in Many-Body Quantum Theory in Condensed Matter Physics: An Introduction (Oxford University Press, Oxford, 2004), pp. 152-183. 